\title{
A rare regulatory variant in the $M E F 2 D$ gene affects gene regulation and splicing and is associated with a SLE sub-phenotype in Swedish cohorts
}

\author{
Fabiana H. G. Farias ${ }^{1,12}$ - Johanna Dahlqvist ${ }^{1}$ - Sergey V. Kozyrev ${ }^{1}$ - Dag Leonard ${ }^{2}$ - Maria Wilbe ${ }^{3,13}$ • \\ Sergei N. Abramov ${ }^{1,4}$ - Andrei Alexsson ${ }^{2}$ - Gerli R. Pielberg ${ }^{1} \cdot$ Helene Hansson-Hamlin ${ }^{5} \cdot$ Göran Andersson $^{3}$. \\ Karolina Tandre ${ }^{2} \cdot$ Anders A. Bengtsson $^{6} \cdot{\text { Christopher Sjöwall }{ }^{7}{ }^{7} \text { - Elisabet Svenungsson }}^{8} \cdot$ Iva Gunnarsson $^{8}$. \\ Solbritt Rantapää-Dahlqvist ${ }^{9} \cdot$ Ann-Christine Syvänen ${ }^{2,10}$ - Johanna K. Sandling ${ }^{2}$. Maija-Leena Eloranta ${ }^{2}$. \\ Lars Rönnblom ${ }^{2} \cdot$ Kerstin Lindblad-Toh ${ }^{1,11}$
}

Received: 28 April 2018 / Revised: 26 August 2018 / Accepted: 9 October 2018 / Published online: 20 November 2018

(c) The Author(s) 2018. This article is published with open access

\begin{abstract}
Systemic lupus erythematosus (SLE) is an autoimmune disorder with heterogeneous clinical presentation and complex etiology involving the interplay between genetic, epigenetic, environmental and hormonal factors. Many common SNPs identified by genome wide-association studies (GWAS) explain only a small part of the disease heritability suggesting the contribution from rare genetic variants, undetectable in GWAS, and complex epistatic interactions. Using targeted resequencing of coding and conserved regulatory regions within and around 215 candidate genes selected on the basis of their known role in autoimmunity and genes associated with canine immune-mediated diseases, we identified a rare regulatory variant rs200395694:G $>\mathrm{T}$ located in intron 4 of the $M E F 2 D$ gene encoding the myocyte-specific enhancer factor 2D transcription factor and associated with SLE in Swedish cohorts (504 SLE patients and 839 healthy controls, $p=0.014$, $\mathrm{CI}=1.1-10)$. Fisher's exact test revealed an association between the genetic variant and a triad of disease manifestations including Raynaud, anti-U1-ribonucleoprotein (anti-RNP), and anti-Smith (anti-Sm) antibodies $(p=0.00037)$ among the patients. The DNA-binding activity of the allele was further studied by EMSA, reporter assays, and minigenes. The region has properties of an active cell-specific enhancer, differentially affected by the alleles of rs200395694:G > T. In addition, the risk allele exerts an inhibitory effect on the splicing of the alternative tissue-specific isoform, and thus may modify the target gene set regulated by this isoform. These findings emphasize the potential of dissecting traits of complex diseases and correlating them with rare risk alleles with strong biological effects.
\end{abstract}

These authors contributed equally: Johanna Dahlqvist, Sergey V. Kozyrev, Dag Leonard, Maria Wilbe

Electronic supplementary material The online version of this article (https://doi.org/10.1038/s41431-018-0297-x) contains supplementary material, which is available to authorized users.

Fabiana H. G. Farias

ffarias@wustl.edu

Kerstin Lindblad-Toh

kersli@broadinstitute.org

Extended author information available on the last page of the article

\section{Introduction}

Systemic lupus erythematosus (SLE) is a chronic inflammatory autoimmune disease that predominantly affects women of childbearing age [1]. A number of studies exploring the genetic basis of SLE in diverse populations identified over 40 risk loci [2], however it was estimated that these loci explain only about $30 \%$ of SLE heritability [3], indicating that disease pathogenesis results from a combined effect of different mechanisms and even a larger number of genes. The recently proposed omnigenic model of complex traits suggests that virtually any gene with regulatory variants active in relevant tissue may contribute to disease pathogenesis [4]. Genes are highly interconnected within the cell-specific gene networks, and thus any effect on one gene with regulatory function, that is not even 
directly related to disease pathways, would lead to waves of perturbations in other genes that would result in increased disease susceptibility. This implies that many non-canonical susceptibility genes remain to be discovered.

Apart from humans, SLE-like disorders have been studied in mice [5] and dogs [6]. These studies revealed potentially shared disease mechanisms across species. We have previously identified five loci associated with a SLErelated disease complex in dogs [7], leading to the detection of a risk haplotype that affects the expression of the BANKI gene [8], which is also associated with human SLE [9] and cause perturbations in B-cell signaling pathways in mice [10]. Identification of shared mechanisms and genes between human and animal diseases could further improve our understanding of SLE.

While GWAS lacks the capability of identifying rare genetic variants, next generation sequencing provides better resolution for discovery of such variants. Here, we used targeted enrichment and high-throughput sequencing of genes from pathways relevant for human immunity, SLEassociated genes, and genes associated with the canine SLErelated disease. Our approach revealed an association of a rare regulatory variant with SLE in the Swedish population.

\section{Materials and methods}

\section{Cases and controls}

To detect novel disease-associated rare variants, we performed targeted re-sequencing of 17 healthy controls and 156 patients; 16 of these had medical record data indicating that they or their parents were born in another country than Sweden (the remaining hereafter referred to as "Swedish"). All patients fulfilled four or more of the American College of Rheumatology (ACR) classification criteria for SLE $[11,12]$ and were enrolled at the outpatient rheumatology clinic at Uppsala University Hospital, Sweden. Clinical data, including age, sex, disease duration, smoking habits, information of ACR, and systemic lupus international collaborating clinics (SLICC) classification criteria [13, 14], SLICC/ACR damage index (SLICC DI), major cardiovascular event (MCE; myocardial infarction, stroke or transient ischemic attack) and Raynaud, as well as results of autoantibody analyses, were collected from medical records. A summary of patient characteristics is presented in Supplementary Table 1. An additional cohort of 364 Swedish SLE cases (average age $=51,84 \%$ females) was used for variant validation by genotyping and for further genetic analysis. A total of 837 healthy blood donors from Uppsala Bioresource (Uppsala, Sweden) matched for age and sex (average age = $50,88 \%$ females) were used as controls. All patients and controls gave their informed consent. The regional ethics committee at Uppsala, Sweden approved the study protocols EPN Uppsala Dnr 00-227 and Dnr 2016/155.

\section{Gene array capture and sequencing data analysis}

For all 215 genes (Supplementary Table 2) chosen for targeted re-sequencing, the following regions were included: all annotated coding exons, 5' UTRs, $3^{\prime}$ UTRs and all conserved elements with a SiPhy [15] lodscore of $>7.5$ based on 29 mammals alignment [16], and located within $100 \mathrm{~kb} 5^{\prime}$ and $3^{\prime}$ of the genes as well as introns. The tiling array comprised 5,059,619 bp in total. Detailed description of array design and sequence analysis is in Supplementary Methods. DNA samples from patients were allocated into ten pools (Supplementary Table 3) and DNA from 17 healthy controls was pooled together. Paired-end sequencing was performed using Illumina HighSeq2000 at the SNP\&SEQ Technology Platform (National Genomics infrastructure, SciLifeLab, Uppsala, Sweden), yielding 100 bp reads. Sequence data was submitted to European Nucleotide Archive (ENA) http://www.ebi.ac.uk/ena/data/ view/PRJEB8904 (study accession number: PRJEB8904).

\section{Variant validation and genetic analysis}

Variants were selected based on a series of filters with strict cutoff thresholds and functional evidence based on ENCODE data and Phylo P scores (Fig. 1). Genotyping by pyrosequencing was used to validate candidate SNPs $(n=10)$ and to identify individuals carrying the variants in the primary patient cohort and 96 Swedish healthy blood donors. Genotyping of the additional cases and controls was performed either by ABI TaqMan allelic discrimination assay on the ABI 7900HT system (Applied Biosystems) or Sanger sequencing. Fisher's exact test was used to analyze associations between 3 candidate SNPs and disease status. The three associated variants were tested for HWE: rs200395694:G > T $p$-value $0.91 ; \quad$ rs867059436:G $>$ A $p$-value $0.98 ;$ rs $576275580: \mathrm{G}>\mathrm{A} p$-value 0.88 .

\section{Reporter assays and EMSA}

The allelic effects were studied using electrophoretic mobility shift assays (EMSA) and luciferase reporter assays. For detailed description see Supplementary methods.

\section{Minigenes and splicing analysis}

The MEF2D minigene constructs containing different allelic variants of rs200395694 were prepared as follows: one intact $2.9 \mathrm{~kb}$ region (positions: chr1:156,479,713-156,482,610; 


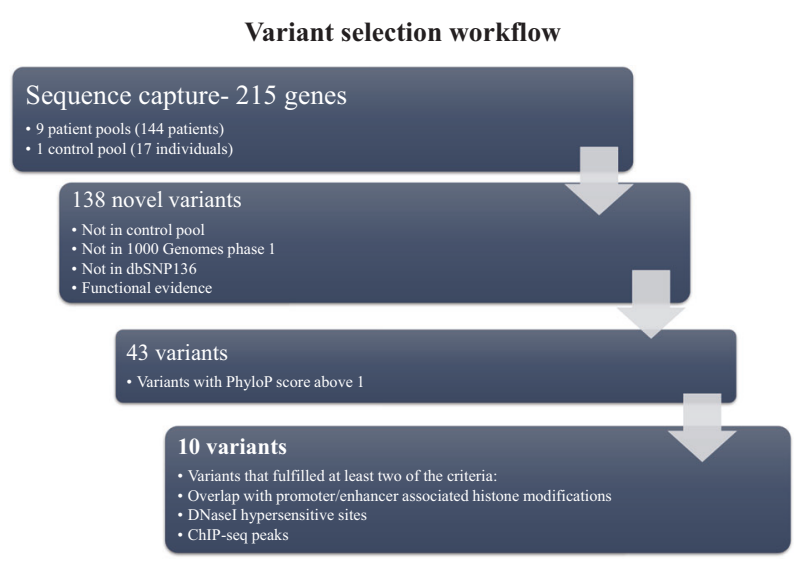

Fig. 1 Flowchart of variant selection. Variant selection was based on a series of filters to remove variants without enough evidence for regulatory potential

hg38) containing four exons including two alternative exons $\alpha 1$ and $\alpha 2$ [17] together with introns was amplified by PCR from a DNA sample with known heterozygous genotype and cloned into pcDNA3.1 D/V5-His -TOPO vector (Invitrogen). The resulting plasmids were validated by Sanger sequencing and purified with EndoFree Plasmid Maxi kit (Qiagen) for transfection experiments. The minigenes were tested in Jurkat, THP-1, HEK293, and C2C12 cell lines using quantitative reverse transcription (RT-PCR) for transcript detection (Supplementary Methods).

\section{Statistical analyses of SLE phenotypes and candidate SNP}

The complete clinical data were available for 140 Swedish patients and these were used for analysis of associations between 21 SLE phenotypes using Fisher's exact test (binary variables) and Mann-Whitney $U$-test (non-binary and ordinal variables). The ACR criterion 10 was removed from genotype-phenotype association analyses as it was well represented by the autoantibodies. Statistical analyses were performed using $\mathrm{R}$. The $p$-value threshold after Bonferroni correction for 21 tests is $2.4 \times 10^{-3}$.

\section{Results}

\section{Targeted re-sequencing and variant selection}

In order to find novel rare variants relevant to SLE pathogenesis, we performed targeted re-sequencing of 215 candidate genes and their potentially regulatory regions including elements highly conserved across mammals [16]. The list of genes comprises known human SLE-associated genes and genes involved in immune response and autoimmunity $(n=77)$, the nuclear factor of activated $\mathrm{T}$ cells (NFAT) pathway genes $(n=98)$, genes in regions associated with dog SLE-like disease [7] and other dog immune-mediated diseases $(n=40) \quad$ (Supplementary Table 2).

We successfully re-sequenced the candidate regions in 140 SLE patients (nine pools) and 17 Swedish healthy individuals (one pool). One of the patient pools containing 4 Swedish and 12 non-Swedish SLE samples failed library preparation and was not sequenced. The average coverage was $3775 \mathrm{X}$ per pool. A summary of the sequencing results can be found in Supplementary Table 3.

A total of 14,206 SNPs were identified in the case pools that were absent in the control pool. To identify novel caseonly variants, all SNPs found only in the case pools were also compared against the 1000 Genomes database (1000G phase 1) and dbSNP136 [18] and only novel SNPs were kept. Later, however, our key SNP was found in dbSNP137. A series of filters with strict cutoff thresholds (Fig. 1) were further applied to select variants with the most evidence for potential regulatory function. Ten SNPs located in six genes fulfilled our criteria by combining strong signals for regulatory potential and were kept for further validation (Supplementary Table 4).

\section{Genotyping and genetic analysis of candidate variants}

Since the variant discovery was performed on pooled DNA, we genotyped our patient cohort in order to identify the individuals carrying the alternative alleles of the ten selected SNPs. In addition, a small control group of 96 Swedish healthy blood donors was also genotyped for comparison. After genotyping, we excluded seven SNPs based on their occurrence in the control group or presence in only one patient. The SNPs that remained were the following: rs200395694:G > T (hg19 chr1:g.156450591 G > T), located in the myocyte enhancer factor 2D $(M E F 2 D)$ gene, rs867059436:G > A (hg19 chr14:g.22958952 G > A), located in the T-cell receptor alpha (TCRA) gene locus, and rs576275580:G > A (hg19 chr15:g.89437973 G > A) located in the first intron of the hyaluronan and proteoglycan link protein 3 (HAPLN3) gene.

The three SNPs were further genotyped in an additional set of 364 Swedish SLE cases and 741 control samples and used for genetic association analysis. Combining all genotyped cases and controls we observed a significant association with SLE only for the $M E F 2 D$ variant rs200395694 $(p=0.014)$. There were in total 12 heterozygotes out of 504 patients and 6 heterozygotes out of 839 controls giving an allele frequency of 0.011 and 0.003 , respectively. The SNP is present in an updated version of dbSNP, and the allele frequency in $1000 \mathrm{G}$ project is 0.001 . The allele frequency in 


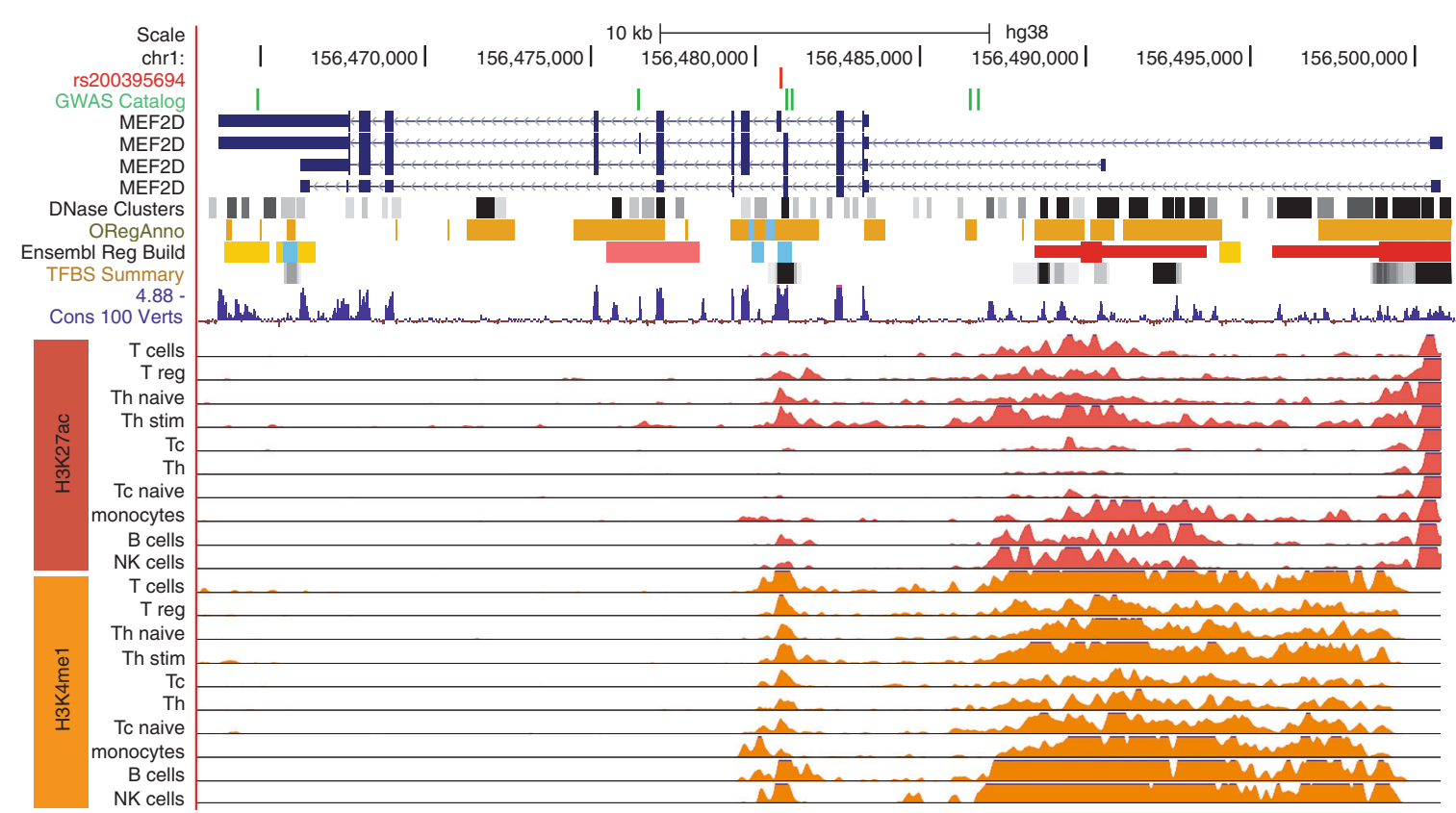

Fig. 2 Functional annotation of the $M E F 2 D$ region with SNP rs200395694. The variant is located in intron 4 of the $M E F 2 D$ gene and overlaps with strong regulatory marks including DNase I hypersensitivity region (DNase Clusters), open regulatory region associated with active gene expression (ORegAnno), conserved transcription factor binding sites (TFBS Summary) and region conservation across 100 vertebrates (Cons 100 Verts) (http://genome.ucsc.edu/). The histone modification marks (H3K27ac, H3K4me1) associated with active enhancers mapped for blood cell populations according to Roadmap Epigenomics (http://egg2.wustl.edu/roadmap/web_portal/) indicate the presence of a cell type-specific enhancer. The known GWA signals located in the MEF2D gene region for migraine and blood cell traits are shown by green vertical lines (GWAS Catalog) [31-33]

healthy controls observed in our study is similar to those reported for the general Swedish population, according to SweFreq (MAF 0.002) (https://swegen-exac.nbis.se/) [19]. Only two alternative alleles were detected in the Swedish population. Of note, there are only heterozygotes reported in both $1000 \mathrm{G}$ and SweFreq, and not a single homozygote was identified for the alternative allele. The other two SNPs were not associated in the larger cohort (HAPLN3: $p=$ 0.818; TCRA: $p=0.188$, Supplementary Table 5).

\section{Functional effects of candidate variant}

The associated SNP rs200395694 is located in the small intron 4 of the myocyte enhancer factor 2D $(M E F 2 D)$ gene (Ensembl:ENSG00000116604) (encoded on the negative strand: ref C, alt A) and overlaps with a DNase I hypersensitive site, histone modifications and transcription factor binding sites (Fig. 2). The PhyloP score of 4.2 indicates high conservation among mammals and two regulatory motifs for Elk1 and GABP transcription factors overlap with the variant (http://genome.ucsc.edu/). The region also contains chromatin modification marks for enhancers active in T, B, NK cells, and monocytes (Fig. 2) (http://www.roa dmapepigenomics.org) [20]. The Regulome database annotates the variant as likely to affecting binding with the score 2a (http://regulomedb.org/snp/chr1/156450590) [21]. Further analyses using TRANSFAC [22] and TRAP [23], indicated stronger binding affinities to the alternative allele for several transcription factors (Supplementary Table 6).

The potential for protein-binding of rs200395694 was first investigated by EMSA using nuclear extracts from Jurkat T-cell line to confirm the Roadmap T-cell-specific enhancer activity and tested also in Daudi B-cell line. We observed differential protein-binding between the reference and the alternative allele in nuclear extracts from both cell lines (illustrated with Jurkat $\mathrm{T}$ cells; Fig. 3a). Next, we explored the regulatory effect of the SNP by luciferase reporter assays. The opposite effect of the alternative allele A on the reporter gene expression in different cells and under different conditions was detected. Thus, the alternative $A$ allele induced expression in non-stimulated Jurkat (1.3-fold, $p=0.02)$ and K562 cells (1.8-fold, $p=0.0001)$ compared to the reference $\mathrm{C}$ allele (Fig. 3b, Supplementary Fig. 1), while in THP-1 this allele suppressed the reporter, and no significant allelic difference was seen in HeLa and Daudi. Interestingly, upon Jurkat stimulation, the reference allele showed much higher luciferase activity (1.5-fold, $p=$ 0.0001 ), suggesting active regulation by another transcription factor(s) with increased binding affinity to the $\mathrm{C}$ allele. In stimulated K562, the luciferase expression driven by the 

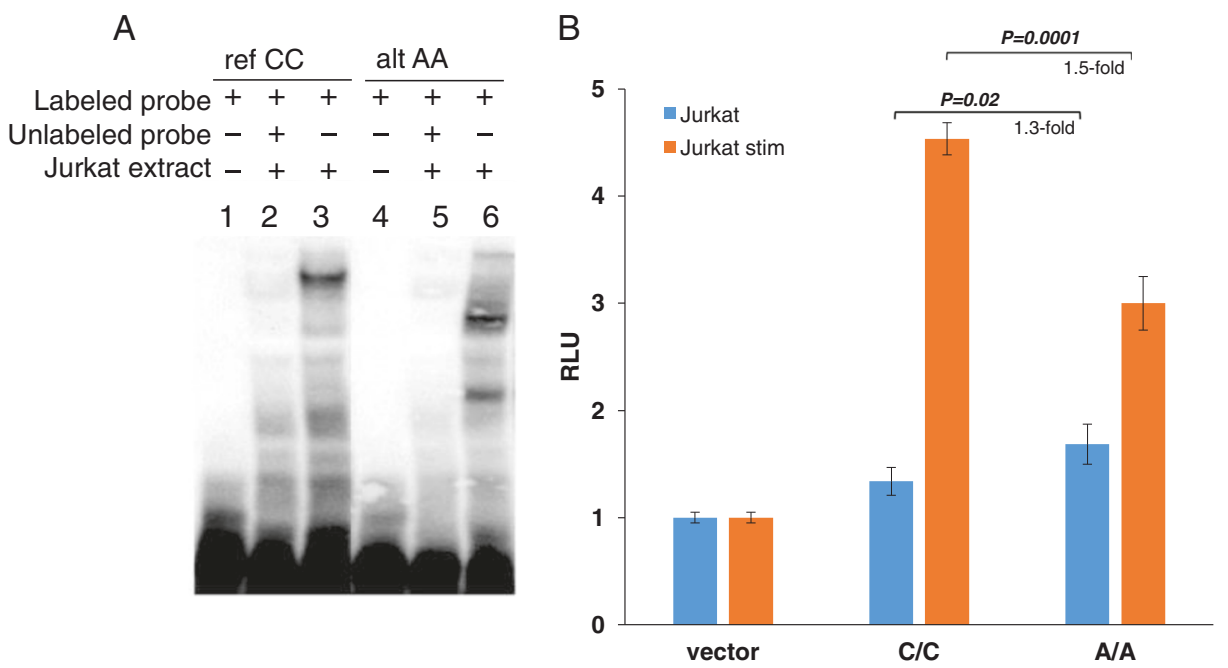

Fig. 3 Binding and regulatory potential of rs200395694 alleles. a EMSA results with nuclear extract from Jurkat cells. b Luciferase reporter assay performed in Jurkat. Bars represent mean values \pm SD. RLU relative light units. Statistical analysis was done using an unpaired $t$-test

risk allele A remains higher compared to the reference allele C (1.4-fold, $p=0.0001$ ) (Supplementary Fig. 1).

Given the reported alternative tissue-specific splicing of the exon located 22 bp downstream of rs200395694 [17, 24] we also studied the potential effect of the rare allele A on splicing using minigenes (Fig. 4a). Both isoforms are expressed in human peripheral blood mononuclear cells (PBMC), monocytic cells THP-1, myelogenous leukemia cells K562, B-cell line Daudi, and murine myoblasts $\mathrm{C} 2 \mathrm{C} 12$, but only the major $\alpha 1$ transcript was detected in Jurkat, cervical cancer cells HeLa and human embryonic kidney cells HEK293 (Supplementary Fig. 2). The minigene constructs with different genotypes were transfected into two cell lines, not expressing the $\alpha 2$ isoform, Jurkat and HEK293, and two lines with detected expression of $\alpha 2$, THP-1, and C2C12 cells. The latter cell line was chosen as a control, since the splicing of the $M E F 2 D$ isoforms has previously been thoroughly examined in $\mathrm{C} 2 \mathrm{C} 12$ cells [17]. No allelic difference was detected for the $\alpha 1$ isoform transcribed from the minigenes in THP-1, HEK293 and Jurkat, and only a marginally significant increase was detected in the A allele-containing constructs transfected in $\mathrm{C} 2 \mathrm{C} 12$ cells (Fig. 4b-e and Supplementary Fig. 3). The alternative isoform $\alpha 2$ generated from the minigene with the rare A allele was significantly repressed in all tested cells. Both isoforms were downregulated upon stimulation of Jurkat and THP-1, while in $\mathrm{C} 2 \mathrm{C} 12$ the major $\alpha 1$ isoform was also downregulated upon cell differentiation, and the minor $\alpha 2$ isoform was upregulated in the reference allele and remained inhibited in the rare A allele (Fig. 4). The downregulation of $\alpha 1$ and induction of $\alpha 2$ upon cell $\mathrm{C} 2 \mathrm{C} 12$ differentiation is in agreement with the previously reported results [17].

\section{Association analysis of the candidate variant with clinical manifestations}

Finally, we investigated whether the rs200395694 variant was specifically associated with any of the clinical phenotypes within the SLE cohort (140 SLE Swedish patients from the discovery set for which we have close to complete clinical information). The clinical characteristics for all patients and for the patients carrying the alternative allele for the candidate SNP are presented in Table 1. We observed a nominally significant association between the $M E F 2 D$ rs200395694-A allele and presence of anti-Sm antibodies $(p=0.0058)$ and anti-RNP antibodies $(p=$ 0.017) with Fisher's exact test. Previous autoantibody profile analyses have shown that anti-RNP and anti-Sm antibodies cluster together in SLE cohorts and that anti-RNP antibodies are associated with Raynaud's phenomenon [25]. In our data, all three individuals carrying the $M E F 2 D$ rs200395694-A allele were positive for these three phenotypes, as compared to 11 individuals $(8.5 \%)$ of all the patients, with a significant association between this triad of disease manifestations and the rs200395694-A allele ( $p=$ 0.00037; Fisher's exact test).

\section{Discussion}

SLE is considered as the prototype of complex autoimmune diseases with significant contribution from genetic background. In an attempt to map loci associated with the disease, numerous GWA studies have been performed in diverse populations (reviewed in ref. [2]). The main drawback of such studies is the inability to detect genetic effects 
A

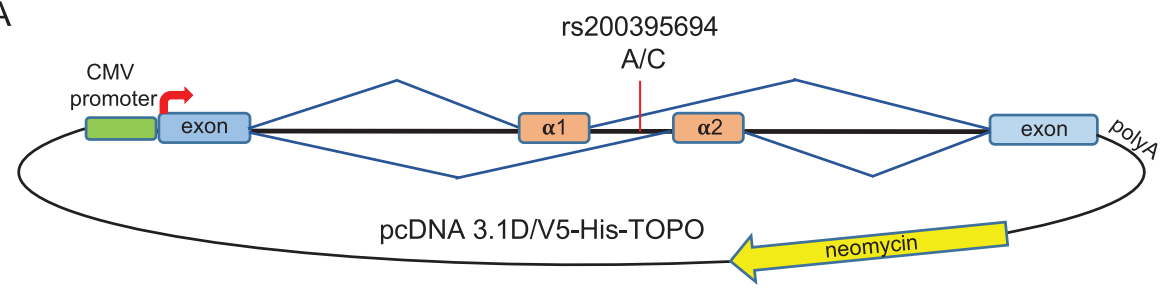

B

THP-1 $\alpha 1$ isoform
-THP-1

- THP-1 stim

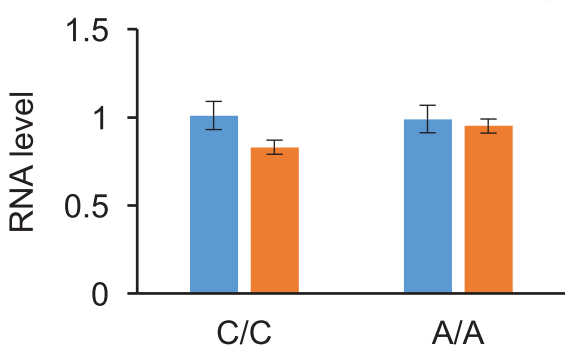

C

THP-1 $\alpha 2$ isoform

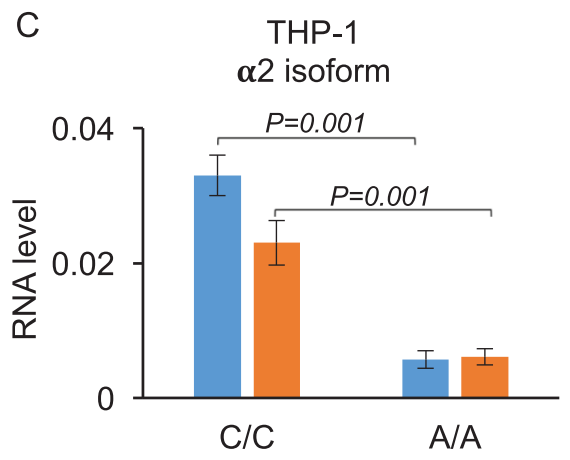

D

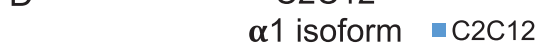

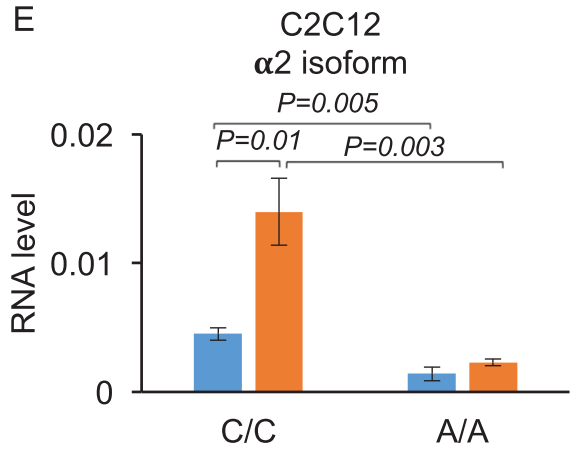

Fig. 4 Analysis of alternative splicing with minigenes. a Minigenes with alternative alleles were cloned into pcDNA3.1 vector between the CMV promoter and the polyadenylation site. Neomycin gene was used for transfection normalization. b-e Levels of alternative isoforms transcribed from minigenes transfected into THP-1 cells $(\mathbf{b}, \mathbf{c})$ and $\mathrm{C} 2 \mathrm{C} 12$ cells $(\mathbf{d}, \mathbf{e})$ were measured by quantitative RT-PCR. THP-1 cells were stimulated with $100 \mathrm{ng} / \mathrm{ml}$ of LPS and $10 \mathrm{ng} / \mathrm{ml}$ of interferon gamma for $12 \mathrm{~h}$. C2C12 cells were differentiated with $2 \%$ horse serum for $64 \mathrm{~h}$. Bars represent mean values \pm SEM

from rare and low frequency variants that may have direct causal effects on genes. While whole-genome sequencing is still a demanding experiment, the use of targeted sequencing of specific regulatory regions in selected genes could be a strategy of choice when analyzing large patient cohorts. As a proof of principle, we focused on coding and conserved regulatory regions in 215 genes selected for their role in immune pathways or for being previously associated with SLE in humans or dogs.

The power to detect rare disease-associated variants depends on the ability to study specifically variants that are likely to have functional consequence. At present, it is much easier to assign potential detrimental function to coding variants. However, based on common variants associated with common diseases (majority of GWA studies signals reside outside coding regions [26]), it is likely that also non-coding variants are involved in the disease pathogenesis and that they can have both low and high effect sizes. For example, for a scenario where selection is relatively weak $\left(s=10^{-3}\right)$ we would need 260 cases to detect an allele with and odds ratio of 20 , while a variant with a twofold increased risk would require 28,000 cases [27]. In this study, we attempted to hedge our bets for discovery of novel rare variants affecting SLE in multiple ways, we: (1) targeted genes in pathways associated to SLE in humans or dogs, (2) targeted evolutionarily conserved non-coding elements in addition to coding regions of these genes, (3) we used multiple functional genomics data sets to point out variants with candidate functions, and (4) looked for novel variants not present in controls and available databases, thus increasing the likelihood that the detected variants would be detrimental. Based on this we were able to find one variant that was both functional and associated to disease subphenotypes. However, it is worth realizing that the current data set will include a lot of false negatives. 
Table 1 Clinical data of Scandinavian patients and patients carrying candidate SNPs in $M E F 2 D$

\begin{tabular}{|c|c|c|c|}
\hline & $\begin{array}{l}\text { Swedish } \\
\text { patients (140) }\end{array}$ & $\begin{array}{l}M E F 2 D \\
\text { rs } 200395694-\mathrm{A} \\
(n=3)\end{array}$ & $p$-value \\
\hline Females/males & $125 / 15$ & $2 / 1$ & \\
\hline Age, mean (range) & $48(20-85)$ & $47(39-51)$ & \\
\hline $\begin{array}{l}\text { Disease duration, mean } \\
\text { (range) }\end{array}$ & $17(0-63)$ & $17(8-27)$ & \\
\hline Smoking (ever), no. (\%) & $50(36)$ & $2(67)$ & \\
\hline \multicolumn{4}{|c|}{ ACR criteria (1982), no. (\%) } \\
\hline 1. Malar rash & $91(65)$ & $2(67)$ & 1 \\
\hline 2. Discoid rash & $43(31)$ & $1(33)$ & 1 \\
\hline 3. Photosensitivity & $97(69)$ & $2(67)$ & 1 \\
\hline 4. Oral ulcers & $41(29)$ & $1(33)$ & 1 \\
\hline 5. Arthritis & $101(72)$ & $3(100)$ & 0.56 \\
\hline 6. Serositis & $50(36)$ & $2(67)$ & 1 \\
\hline 7. Renal disorder & $33(24)$ & $1(33)$ & 0.557 \\
\hline 8. Neurologic disorder & $7(5)$ & 0 & 1 \\
\hline $\begin{array}{l}\text { 9. Hematologic } \\
\text { disorder }\end{array}$ & $87(62)$ & $3(100)$ & 0.289 \\
\hline $\begin{array}{l}\text { 10. Immunologic } \\
\text { disorder }\end{array}$ & $87(62)$ & $3(100)$ & - \\
\hline $\begin{array}{l}\text { 11. Anti-nuclear } \\
\text { antibodies }\end{array}$ & 137 (98) & $3(100)$ & 1 \\
\hline $\begin{array}{l}\text { Total ACR criteria, } \\
\text { median (range) }\end{array}$ & $5(4-9)$ & $7(5-8)$ & 0.179 \\
\hline $\begin{array}{l}\text { SLICC DI, median } \\
\text { (range) }\end{array}$ & $0(0-6)$ & $2(0-3)$ & 0.202 \\
\hline \multicolumn{4}{|l|}{ Presence of, no. (\%) } \\
\hline Anti-dsDNA antibodies & $82(59)$ & $3(100)$ & 0.267 \\
\hline Anti-Sm antibodies & $26(19)$ & $3(100)$ & 0.0058 \\
\hline Anti-RNP antibodies & $37(26)$ & $3(100)$ & 0.017 \\
\hline Anti-SSA antibodies & $67(48)$ & $1(33)$ & 1 \\
\hline Anti-SSB antibodies & $31(22)$ & $2(67)$ & 0.123 \\
\hline $\begin{array}{l}\text { Anti-cardiolipin } \\
\text { antibodies (IgM or IgG) }\end{array}$ & $52(39)$ & $1(33)$ & 1 \\
\hline $\begin{array}{l}\text { Major cardiovascular } \\
\text { event }\end{array}$ & $16(11)$ & $1(33)$ & 0.307 \\
\hline Raynaud & $64(50)$ & $3(100)$ & 0.244 \\
\hline $\begin{array}{l}\text { Raynaud, anti-RNP and } \\
\text { anti-Sm antibodies }\end{array}$ & $11(8.7)$ & $3(100)$ & 0.00037 \\
\hline
\end{tabular}

$p$-values were calculated using Fisher's exact test and Mann-Whitney $U$-test. ACR criterion 10 (Immunologic disorder) was excluded from the association analyses as it was strongly correlated with presence of anti-dsDNA, anti-RNP, and anti-Sm. Missing data $(n)$ for Raynaud: 13 ; Anti-cardiolipin antibodies: 5; Smoking: 1; remaining parameters: 0

ACR American College of Rheumatology, SLICC Systemic Lupus International Collaborating Clinics, SLICC DI SLICC damage index, Age age at time of data collection, Major cardiovascular event transient ischemic attack, stroke, or myocardial infarction

We identified a rare variant located in intron 4 of $M E F 2 D$ gene significantly enriched in Swedish SLE patients. This gene was selected for targeted sequencing based on its role in the NFAT pathway. The genetic association signals in the NFAT pathway genes with SLE were identified in dogs [7], thereby making canine associated genes and pathways valuable candidates for investigation in human disease. $M E F 2 D$ encodes for a member of the myocyte enhancer factor 2 family of transcription factors, widely expressed in different tissues, including all hematopoietic cell populations [28]. MEF2D is an essential transcriptional activator of the interleukin $2(I L-2)$ gene [29]. The dysregulation of IL-2 production is a common characteristic of $\mathrm{T}$ cells in SLE [30]. Interestingly, several common SNPs in the $M E F 2 D$ gene have been reported to be strongly associated with migraine [31, 32] and blood cell phenotypes [33], but none of them have been implicated in association with any autoimmune disease.

The A allele of the MEF2D SNP rs200395694 was also significantly associated with the triad of disease manifestations comprising of anti-Sm, anti-RNP antibodies, and Raynaud's phenomenon in our patient cohort. The association between anti-RNP and anti-Sm antibodies to Raynaud's phenomenon has been previously established [25], implying a common mechanism involved in the etiology of these manifestations; nonetheless to our knowledge, no gene has been associated with this combined phenotype to date.

The combined evidence from genomic annotations suggested the presence of an active cell type-specific enhancer in the region harboring the SNP. This is fully supported by the results obtained in our in vitro experiments, where they indicate that both alleles bind specifically to protein complexes, but that these are different for the two alleles. This suggests that different transcription factors may recognize the fragment depending on the allele present and eventually participate in gene regulation (Fig. 3a). The luciferase reporter assay confirmed the cell line-specific enhancer with significant differential allelic effect: the two alleles had different levels of expression for both unstimulated and stimulated Jurkat cells (Fig. 3b and Supplementary Fig. 1).

The $M E F 2 D$ gene produces two alternatively spliced transcripts $[17,24]$ generating the ubiquitous $\alpha 1$ isoform and the inducible tissue-specific $\alpha 2$ isoform. Previously, the $\alpha 2$ transcript was detected only in muscle tissue and was studied in the murine myoblast cells $\mathrm{C} 2 \mathrm{C} 12$ in more detail [17]. We detected low levels of the $\alpha 2$ isoform in human PBMC, as well as in THP-1, K562 and Daudi cell lines, which may suggest a putative role of the isoform in these cells. Interestingly, we found that the novel risk allele inhibits splicing of the $\alpha 2$ isoform transcribed from the minigene, while not affecting the $\alpha 1$ isoform.

The $\alpha 2$ isoform overexpressed in $\mathrm{C} 2 \mathrm{C} 12$ cells regulated expression of a specific set of genes, only partially overlapping with those controlled by the $\alpha 1$ isoform [17]. Of note, two of the genes, specifically induced only by the $\alpha 2$ isoform, were DNASEIL3 and AIM2, both previously shown to be involved in SLE pathogenesis [34-37]. The role of the $\alpha 2$ isoform in immune cells remains unclear at this stage, but in the light of our current results it certainly requires a comprehensive investigation. We do not know if 
the cell-specific enhancer with rs200395694 that presumably affects $M E F 2 D$ expression could have an effect on $\alpha 2$ cell type-specific splicing, or if these two events are unrelated. For instance, in T cells, that do not express the $\alpha 2$ isoform, the main outcome of the risk allele would be on the regulation of gene expression. On the other hand, in monocytes normally producing the $\alpha 2$ isoform, the inhibition of it would have an effect on the pattern of the downstream target genes. It is tempting to speculate, for example, that direct correlation between the expression of the $\alpha 2$ transcript and DNASE1L3 would result in suppression of DNASE1L3 in monocytes in the risk genotype where the $\alpha 2$ is inhibited. The loss-of-function of DNASE1L3 causes severe familial SLE [38].

In summary, in search for rare disease variants, usually missed in GWA studies, we used targeted re-sequencing of regulatory highly conserved regions in selected genes in a well-characterized cohort of SLE patients. We present evidence of genetic association of a rare variant rs200395694: $\mathrm{G}>\mathrm{T}$ with SLE in Swedish patients. The risk allele is associated also with the triad of disease manifestations comprised of anti-Sm, anti-RNP antibodies, and Raynaud's phenomenon. The SNP rs200395694:G > T is located in a cell type-specific enhancer and appears to influence expression and splicing of the $M E F 2 D$ mRNA. While $M E F 2 D$ is a widely expressed transcription factor, finetuning of its transcription and splicing in relevant cells may have an effect on downstream target genes and may contribute to the disease pathogenesis. We believe that our results support the omnigenic model for complex traits where $M E F 2 D$ shows a rather modest association with SLE in our cohort of Swedish patients but apparent functional effect. Therefore, $M E F 2 D$ could be considered as a peripheral gene for SLE.

Acknowledgements We thank Rezvan Kiani Dehkordi for the collection of SLE blood samples. We also gratefully acknowledge Eva Freyhult at the Bioinformatics Short-Term Support and Infrastructure platform at Science for Life Laboratory, Uppsala for help with statistical analyses. The SNP\&SEQ Technology Platform at Science for Life Laboratory, Uppsala performed sequencing and analysis was performed using the computational resources at UPPNEX. All three platforms are part of Science for Life Laboratory at Uppsala University and supported as a national infrastructure by the Swedish Research Council.

Funding This work was supported by the Knut and Alice Wallenberg Foundation; Swedish Research Council; Swedish Research Council FORMAS; Swedish Rheumatism Foundation; and King Gustaf V's 80-Year Foundation. FHGF is a recipient of a Swedish Institute scholarship and JD of a King Gustav V 80th year's Foundation scholarship. SNA was supported within the frame of the program of competitive growth of Kazan Federal University subsidized by the Russian Government. KLT is the recipient of an ERC Consolidator Award and is a Wallenberg Scholar. DL and JD are recipients of the Agnes and Mac Rudberg Foundation Access to Learning Fund from Uppsala County Council and Uppsala University Hospital.

\section{Compliance with ethical standards}

Conflict of interest The authors declare that they have no conflict of interest.

Open Access This article is licensed under a Creative Commons Attribution 4.0 International License, which permits use, sharing, adaptation, distribution and reproduction in any medium or format, as long as you give appropriate credit to the original author(s) and the source, provide a link to the Creative Commons license, and indicate if changes were made. The images or other third party material in this article are included in the article's Creative Commons license, unless indicated otherwise in a credit line to the material. If material is not included in the article's Creative Commons license and your intended use is not permitted by statutory regulation or exceeds the permitted use, you will need to obtain permission directly from the copyright holder. To view a copy of this license, visit http://creativecommons. org/licenses/by/4.0/.

\section{References}

1. Tsokos GC. Systemic lupus erythematosus. N Engl J Med. 2011;365:2110-21.

2. Tsokos GC, Lo MS, Costa Reis P, Sullivan KE. New insights into the immunopathogenesis of systemic lupus erythematosus. Nat Rev Rheumatol. 2016;12:716-30.

3. Morris DL, Sheng Y, Zhang Y, Vyse TJ. Genome-wide association meta-analysis in Chinese and European individuals identifies ten new loci associated with systemic lupus erythematosus. Nat Genet. 2016;48:940-6.

4. Boyle EA, Li YI, Pritchard JK. An expanded view of complex traits: From polygenic to omnigenic. Cell . 2017;169:1177-86.

5. Perry D, Sang A, Yin Y, Zheng Y, Morel L. Murine models of systemic lupus erythematosus. J Biomed Biotechnol. 2011;2011:271694.

6. Hansson-Hamlin H, Lilliehook I. A possible systemic rheumatic disorder in the Nova Scotia duck tolling retriever. Acta Vet Scand. 2009;51:16.

7. Wilbe M, Jokinen P, Truvé K, Seppala EH, Karlsson EK, Biagi T, et al. Genome-wide association mapping identifies multiple loci for a canine SLE-related disease complex. Nat Genet. 2010;42:250-4.

8. Wilbe M, Kozyrev SV, Farias FHG, Bremer HD, Hedlund A, Pielberg GR, et al. Multiple changes of gene expression and function reveal genomic and phenotypic complexity in SLE-like disease. PLoS Genet. 2015;11:e1005248.

9. Kozyrev SV, Abelson AK, Wojcik J, Zaghlool A, Reddy MVPL, Sanchez E, et al. Functional variants in the B-cell gene BANK1 are associated with systemic lupus erythematosus. Nat Genet. 2008;40:211-6.

10. Wu YY, Kumar R, Iida R, Bagavant H, Alarcón-Riquelme ME. BANK1 regulates IgG production in a lupus model by controlling TLR7-dependent STAT1 activation. PLoS ONE 2016;11: e0156302.

11. Tan EM, Cohen AS, Fries JF, Masi AT, Mcshane DJ, Rothfield $\mathrm{NF}$, et al. The 1982 revised criteria for the classification of systemic lupus erythematosus. Arthritis Rheum. 1982;25: 1271-7.

12. Hochberg MC. Updating the American College of Rheumatology revised criteria for the classification of systemic lupus erythematosus. Arthritis Rheum. 1997;40:1725.

13. Gladman D, Ginzler E, Goldsmith C, Fortin P, Liang M, SanchezGuerrero $\mathrm{J}$, et al. The development and initial validation of the Systemic Lupus International Collaborating Clinics/American 
College of Rheumatology damage index for systemic lupus erythematosus. Arthritis Rheum. 1996;39:363-9.

14. Petri, M, Orbai, A-M, Alarcon, GS, Gordon, C, Merrill, JT, Fortin $\mathrm{PR}$, et al. Derivation and validation of the Systemic Lupus International Collaborating Clinics classification criteria for systemic lupus erythematosus. Arthritis Rheum. 2012;64:2677-86.

15. Garber M, Guttman M, Clamp M, Friedman N, Xie X. Identifying novel constrained elements by exploiting biased substitution patterns. Bioinformatics. 2009;25:154-62.

16. Lindblad-Toh K, Garber M, Zuk O, Lin MF, Parker BJ, Washietl $\mathrm{S}$, et al. A high-resolution map of human evolutionary constraint using 29 mammals. Nature. 2011;478:476-82.

17. Sebastian S, Faralli H, Yao Z, Rakopoulos P, Palii C, Cao Y, et al. Tissue-specific splicing of a ubiquitously expressed transcription factor is essential for muscle differentiation. Genes Dev. 2013;27:1247-59.

18. Sherry ST, Ward MH, Kholodov M, Baker J, Phan L, Smigielski EM, et al. dbSNP: the NCBI database of genetic variation. Nucleic Acids Res. 2001;29:308-11.

19. Ameur A, Dahlberg J, Olason P, Vezzi F, Karlsson R, Martin M, et al. SweGen: a whole-genome data resource of genetic variability in a cross-section of the Swedish population. Eur J Hum Genet. 2017;25:1253-60.

20. Roadmap Epigenomics C, Kundaje A, Meuleman W, Ernst J, Bilenky M,Yen A, et al. Integrative analysis of 111 reference human epigenomes. Nature. 2015;518:317-30.

21. Boyle AP, Hong EL, Hariharan M, Cheng Y, Schaub MA, Kasowski M, et al. Annotation of functional variation in personal genomes using RegulomeDB. Genome Res. 2012;22:1790-7.

22. Matys V, Fricke E, Geffers R, Gößling E, Haubrock M, Hehl R, et al. TRANSFAC: transcriptional regulation, from patterns to profiles. Nucleic Acids Res. 2003;31:374-8.

23. Thomas-Chollier M, Hufton A, Heinig M, O'Keeffe S, El Masri $\mathrm{N}$, Roider HG, et al. Transcription factor binding predictions using TRAP for the analysis of ChIP-seq data and regulatory SNPs. Nat Protoc. 2011;6:1860-9.

24. Martin JF, Miano JM, Hustad CM, Copeland NG, JenkinsNA, Olson EN. A Mef2 gene that generates a muscle-specific isoform via alternative mRNA splicing. Mol Cell Biol. 1994;14:1647-56.

25. Hoffman IE, Peene I, Meheus L, Huizinga TWJ, Cebecauer L, Isenberg D, et al. Specific antinuclear antibodies are associated with clinical features in systemic lupus erythematosus. Ann Rheum Dis. 2004;63:1155-8.

26. MacArthur J, Bowler E, Cerezo M, Gil L, Hall P, Hastings E, et al. The new NHGRI-EBI Catalog of published genome-wide association studies (GWAS Catalog). Nucleic Acids Res. 2017;45: D896-901.

27. Zuk O, Schaffner SF, Samocha K, Do R, Hechter E, Kathiresan S, et al. Searching for missing heritability: designing rare variant association studies. Proc Natl Acad Sci USA. 2014;111: E455-64.

28. Breitbart RE, Liang CS, Smoot LB, Laheru DA, Mahdavi V, Nadal-Ginard B, et al. A fourth human MEF2 transcription factor, $\mathrm{hMEF} 2 \mathrm{D}$, is an early marker of the myogenic lineage. Development. 1993;118:1095-106.

29. Pan F, Ye Z, Cheng L, Liu JO. Myocyte enhancer factor 2 mediates calcium-dependent transcription of the interleukin-2 gene in T lymphocytes: a calcium signaling module that is distinct from but collaborates with the nuclear factor of activated $\mathrm{T}$ cells (NFAT). J Biol Chem. 2004;279:14477-80.

30. Moulton VR, Tsokos GC. Abnormalities of T cell signaling in systemic lupus erythematosus. Arthritis Res Ther. 2011;13:207.

31. Freilinger T, Anttila V, de Vries B, Malik R, Kallela M, Terwindt GM, et al. Genome-wide association analysis identifies susceptibility loci for migraine without aura. Nat Genet. 2012;44: 777-82.

32. Gormley P, Anttila V, Winsvold BS, Palta P, Esko T, Pers TH, et al. Meta-analysis of 375,000 individuals identifies 38 susceptibility loci for migraine. Nat Genet. 2016;48:856-66.

33. Astle WJ, Elding H, Jiang T, Allen D, Ruklisa D, Mann AL, et al. The allelic landscape of human blood cell trait variation and links to common complex disease. Cell. 2016;167:1415-29 e19.

34. Zhao Q, Yang C, Wang J, Li Y, Yang P. Serum level of DNase113 in patients with dermatomyositis/polymyositis, systemic lupus erythematosus and rheumatoid arthritis, and its association with disease activity. Clin Exp Med. 2017;17:459-65.

35. Zhang W, Cai Y, Xu W, Yin Z, Gao X, Xiong S. AIM2 facilitates the apoptotic DNA-induced systemic lupus erythematosus via arbitrating macrophage functional maturation. J Clin Immunol. 2013;33:925-37.

36. Zuo X, Sun L, Yin X, Gao J, Sheng Y, Xu J, et al. Whole-exome SNP array identifies 15 new susceptibility loci for psoriasis. Nat Commun. 2015;6:6793.

37. Al-Mayouf SM, Sunker A, Abdwani R, Al Abrawi S, Almurshedi $\mathrm{F}$, Alhashmi N, et al. Loss-of-function variant in DNASE1L3 causes a familial form of systemic lupus erythematosus. Nat Genet. 2011;43:1186-8.

38. Sisirak V, Sally B, D'Agati V, Martinez-Ortiz W, Özçakar ZB, David J, et al. Digestion of chromatin in apoptotic cell microparticles prevents autoimmunity. Cell . 2016;166:88-101.

\section{Affiliations}

Fabiana H. G. Farias ${ }^{1,12,}$ - Johanna Dahlqvist ${ }^{1}$ - Sergey V. Kozyrev ${ }^{1}$ - Dag Leonard ${ }^{2}$ - Maria Wilbe ${ }^{3,13,}$. Sergei N. Abramov ${ }^{1,4} \cdot$ Andrei Alexsson $^{2} \cdot$ Gerli R. Pielberg $^{1} \cdot$ Helene Hansson-Hamlin ${ }^{5} \cdot$ Göran Andersson $^{3}$. Karolina Tandre ${ }^{2}$. Anders A. Bengtsson ${ }^{6}$. Christopher Sjöwall $\mathbb{D}^{7}$ • Elisabet Svenungsson ${ }^{8} \cdot$ Iva Gunnarsson $^{8}$. Solbritt Rantapää-Dahlqvist ${ }^{9} \cdot$ Ann-Christine Syvänen ${ }^{2,10}$ • Johanna K. Sandling ${ }^{2} \cdot$ Maija-Leena Eloranta $^{2}$. Lars Rönnblom ${ }^{2} \cdot$ Kerstin Lindblad-Toh ${ }^{1,11}$

1 Science for Life Laboratory, Department of Medical Biochemistry and Microbiology, Uppsala University, Box 582, SE-751 24 Uppsala, Sweden

2 Science for Life Laboratory, Department of Medical Sciences, Section of Rheumatology, Uppsala University, SE-751 85 Uppsala, Sweden

3 Department of Animal Breeding and Genetics, Swedish University of Agricultural Sciences (SLU), Box 7023, SE-750 07 Uppsala, Sweden
Institute of Fundamental Medicine and Biology, Kazan Federal University, Kazan 420008, Russia

5 Department of Clinical Sciences, Swedish University of Agricultural Sciences (SLU), Box 7054, SE-750 07 Uppsala, Sweden

6 Department of Clinical Sciences Lund, Lund University, Skane University Hospital, SE-221 00 Lund, Sweden 
7 Department of Clinical and Experimental Medicine,

Rheumatology/Division of Neuro and Inflammation Sciences,

Linköping University, SE-581 85 Linköping, Sweden

8 Rheumatology Unit, Department of Medicine, Solna, Karolinska Institutet, Karolinska University Hospital, SE-171 76

Stockholm, Sweden

9 Department of Public Health and Clinical Medicine/ Rheumatology, Umeå University, SE-901 85 Umeå, Sweden

10 Department of Medical Sciences, Molecular Medicine and Science for Life Laboratory, Uppsala University, SE-754 11 Uppsala, Sweden

11 Broad Institute, Cambridge, 7 Cambridge Center, Cambridge, MA 02142, USA

12 Present address: Department of Psychiatry, Washington University School of Medicine, St. Louis, MO 63110, USA

13 Present address: Science for Life Laboratory, Department of Immunology, Genetics and Pathology, Uppsala University, Box 582, SE-751 24 Uppsala, Sweden 